\title{
GERENCIAMENTO DE ESTOQUES E VENDOR MANAGED INVENTORY: UM ESTUDO DE CASO EM UMA REDE SUPERMERCADISTA
}

\author{
L.C.T.Vilhena ${ }^{1}$; P.C.C.Ribeiro ${ }^{2 *}$ \\ 1 Universidade Federal de Ouro Preto, 35400-000, Ouro Preto-MG, Brasil \\ 2 Universidade Federal Fluminense, 24220-900, Niterói-RJ, Brasil \\ * priscillaribeiro@id.uff.br
}

Artigo submetido em 01/2015 e aceito em 04/2015

\section{RESUMO}

Dentre as atividades logísticas, o gerenciamento de estoques se destaca por apresentar custo elevado, ser responsável por absorver flutuações na demanda, e garantir o uso produtivo dos recursos operacionais. Portanto, uma ferramenta que permite uma gestão mais eficiente dos estoques é o Estoque Gerenciado pelo Fornecedor (Vendor Managed Inventory - VMI). Ela possibilita ao fornecedor um acompanhamento do nível de estoque de seus produtos no cliente, sendo de sua responsabilidade o ressuprimento imediato quando o estoque de segurança é atingido. Diante de tal importância, o presente estudo tem por objetivo analisar a adoção do VMI na gestão de estoque de um varejista de alimentos. Para isso, foi utilizada uma abordagem qualitativa, método estudo de caso, com entrevistas semiestruturadas com um roteiro de perguntas. Assim, através da presente pesquisa foi possível identificar os ganhos com o VMI, como custos com estoques e entregas.

PALAVRAS-CHAVE: Estoque gerenciado pelo fornecedor, varejo, supermercado.

\section{STOCK MANAGEMENT AND VENDOR MANAGED INVENTORY: A CASE STUDY IN A SUPERMARKET NETWORK}

\begin{abstract}
Among logistics activities, inventory management stands out for cost increasing, demand fluctuations and for ensuring reasonable operational resources use. Therefore, a tool that enables more efficient inventory management Vendor Managed Inventory - VMI. It enables inventory monitoring by the supplier, being his responsibility immediately resupply when safety stock is reached. In
\end{abstract}

face of such importance, this study aims to analyze VMI adoption in a food retailer inventory. So, a qualitative approach was used, presenting a case study, with semistructured interviews with a script of questions. Thus, through this research we could identify advantages such as stock and delivery cost reduction.

KEYWORDS: Vendor Managed Inventory, retail, supermarket. 


\section{INTRODUÇÃO}

Segundo Braglia et al. (2014), as empresas, para aumentar sua competitividade, passam a ser uma parte integrante da cadeia de suprimentos em lugar de simples entidades, preocupadas, entre outras atividades, com a gestão de estoques. Para Amirjabbari e Bhuiyan (2014), ter um nível de estoques eficiente é um passo em direção ao aumento do turnover do estoque em empresas. De acordo com a definição de turnover, reduzir o nível de estoque auxilia a aumentar o seu giro, embora, o estoque de segurança seja necessário para proteger as empresas das incertezas inerentes às transações de compras e vendas.

O fluxo ou disponibilidade de produtos é uma medida crítica para o desempenho da logística e da cadeia de suprimentos. Há diferentes resultados que causam quebras e indisponibilidade de produtos na cadeia, como por exemplo, a variedade, seja na demanda ou no lead time; qualidade; desempenho insatisfatório nas entregas; programação inadequada; problemas na manutenção; capacidade produtiva inadequada, entre outros (AMIRJABBARI; BHUIYAN, 2014). Essas falhas podem ser eliminadas com o uso correto do Estoque Gerenciado pelo Fornecedor (Vendor Managed Inventory - VMI) suportado por uma colaboração efetiva entre os pares da cadeia de suprimentos. Kraft Foods, Walmart, Barilla, McCain Foods, HP, Shell estão utilizando a ferramenta (SETAK; DANESHFAR, 2014; MARQUÈS et al., 2010).

Ao considerar uma cadeia de suprimentos descentralizada, Chen (2013) afirma que o VMI, via contrato de consignação e compartilhamento de receitas, torna mais produtivo o processo de tomada de decisão, assim como o fluxo de mercadorias e informação. Tendo em vista que o maior problema no gerenciamento de estoques é a acuracidade, o estudo se justifica por buscar no auxílio prestado pela utilização do VMI para o gerenciamento do estoque, estudar e apresentar seu uso da ferramenta nessa atividade logística de um grande varejista de alimentos, identificando seus pontos fortes e a melhorar, sempre buscando o melhor atendimento ao cliente e a redução de custos.

Este artigo tem como objetivo analisar a adoção do VMI na gestão de estoque de um varejista de alimentos. Para suportá-lo, o objetivo secundário é descrever o processo de integração possibilitada pelo VMI na amostra escolhida.

\section{REVISÃO DA LITERATURA}

\subsection{Gestão, Planejamento e Políticas de Estoques: principais aspectos}

Gianesi e Biazzi (2011) definiram três objetivos principais para a gestão de estoques: maximizar o nível de serviço ou o atendimento da demanda pela disponibilidade do material em estoque; maximizar o giro de estoques ou o investimento em estoques e seus custos correspondentes; maximizar a eficiência operacional, reduzindo os custos do processo de suprimento (aquisição, transferência ou produção dos materiais). No entanto, para os dois autores citados acima, os três objetivos são conflitantes, ou seja, a implantação de um objetivo implicará num pior desempenho relacionado aos dois outros. Dessa forma, deve-se saber qual ou quais objetivos serão priorizados e em que medida, que varia de empresa para empresa.

Quanto à gestão de estoques, tem-se: os princípios, os custos e as funcionalidades. Os princípios são os responsáveis por facilitar a correta administração do fator produção-demanda, uma vez que não é possível produzir bens e serviços que atendam de forma constante e instantânea as quantidades requeridas pelos clientes. Nessa atividade, há decisões relacionadas a compras, produção e distribuição, com uma análise de aspectos que permitam o atendimento ao cliente com custos reduzidos. Os aspectos são: quantidade de matéria prima, definição de quando pedir e frequência de revisão, localização de estoques e seu controle (SANTOS, 2009). 
Ballou (2006) afirma que os custos se dividem em três categorias: manutenção, requisição ou compra e falta de estoque. Já as funcionalidades são quatro: especialização geográfica, desacoplamento, equilíbrio entre oferta e demanda e proteção contra incertezas (BOWERSOX et al., 2007).

Em relação ao planejamento, Gianesi e Biazzi (2011) afirmam que os estoques são necessários na medida em que os processos de suprimento e de demanda não podem ser sincronizados de forma que as taxas de demanda e suprimento sejam idênticas em cada instante. Assim, o planejamento, o dimensionamento e a gestão são fatores determinantes no sucesso na disponibilização dos produtos no tempo e na qualidade desejadas pelo cliente. O planejamento pode ser eficiente através de políticas distintas e afins ao estoque, identificando-se dois tipos de estoques: estoques de produtos homogêneos e estoques multi itens. Para o estoque de itens homogêneos a curva $\mathrm{ABC}$ é bastante utilizada, o que resulta num maior controle de estoque. Nos estoques multi itens por haver uma grande variedade de itens armazena-se em estoque e há um aumento considerável na complexidade do gerenciamento, podendo classificá-los como multicritérios. Os critérios podem ser vários: lead time, existência de atributos comuns, obsolescência, facilidade de substituição, escassez, durabilidade, distribuição de demanda, entre outros (SANTOS; RODRIGUES, 2006).

De acordo com Silva et al. (2008), há dois tipos de políticas: o sistema de revisão contínua ou o sistema de revisão periódica. No sistema de revisão contínua os pedidos de reposição de materiais podem ser realizados em qualquer instante, visto que os estoques são continuamente monitorados e sabe-se o nível de estocagem em qualquer momento. Já no sistema de revisão periódica, as ordens são emitidas em intervalos pré-determinados em intervalos de tempo.

Regoa e Mesquita (2011) apresentam que além dos dois sistemas anteriores, existe o sistema de estoque base, que tem como parâmetro a base ou patamar de estoque "B"; a cada retirada do estoque, realiza-se um pedido de igual quantidade para repor a base, mantendo-se a posição do estoque (estoque em mãos mais as ordens em aberto) constante.

Com o alinhamento da gestão, planejamento e política, o gerenciamento do estoque influenciará positivamente no bom funcionamento de toda a cadeia de suprimentos, podendo por muitas vezes alertar para problemas na manufatura, caso seja percebido uma mudança no volume de produto estocado (VASCONCELOS et al., 2011).

No apoio à gestão eficiente dos estoques e da cadeia, Oliveira e Hatakeyama (2012) afirmam que tecnologias de informação como o ERP contribuem para a eficiência das empresas, pois oferecem resultados melhores por meio da administração integrada dos recursos, automatização dos processos e melhor fluxo de informações.

Segundo Bertaglia (2009), as organizações devem definir e manter uma estratégia de estoques, que, conduzida adequadamente, assegurará um balanceamento dos processos de produção e distribuição, minimizando os estoques. Desse modo, ocorrerá o posicionamento estratégico dos produtos, fato que influenciará diretamente na maneira pela qual o estoque será administrado.

\subsection{VMI e a Gestão de estoques: pontos críticos, barreiras, sucesso e fracasso}

Atualmente, o propósito das parcerias é compartilhar a informação entre os membros da cadeia de suprimentos, resultando em lead times reduzidos, estoques menores, obsolescência reduzida e manufatura mais eficiente (SETAK; DANESHFAR, 2014). Sgarbi Junior e Cardoso (2011) afirmam que uma estratégia eficiente de estoques na cadeia de suprimentos pode elevar o nível de serviço ao cliente, reduzir prazos e custos, atender a demanda e obter eficiência financeira. 
Silva (2010) apresenta os fatores críticos, as barreiras, os fatores de sucesso e de fracasso da política do VMI no quadro 1:

Quadro 1 - Funcionamento do VMI

\begin{tabular}{|c|c|c|c|}
\hline Críticos & Barreiras & Sucessos & Fracassos \\
\hline $\begin{array}{l}\text { Compartilhamento, } \\
\text { disponibilidade, } \\
\text { confiabilidade, precisão } \\
\text { facilidade de acesso e } \\
\text { consistência das informações }\end{array}$ & $\begin{array}{l}\text { Falta de preparo em } \\
\text { conectar informações de } \\
\text { demanda ao seu programa } \\
\text { de produção e controle de } \\
\text { inventários }\end{array}$ & $\begin{array}{l}\text { Melhorias dos níveis de } \\
\text { serviço, diminuição de pedidos } \\
\text { emergenciais e erros em } \\
\text { pedidos, avanços no controle } \\
\text { da cadeia e reduções de } \\
\text { inventário. }\end{array}$ & $\begin{array}{l}30-40 \% \text { conseguem } \\
\text { alguns benefícios e } \\
20-30 \% \text { não geram } \\
\text { nenhum resultado }\end{array}$ \\
\hline Confiança & $\begin{array}{l}\text { Falta de processos que } \\
\text { integrem as informações }\end{array}$ & $\begin{array}{l}\text { Melhorias nos níveis de } \\
\text { serviço, depois no controle da } \\
\text { cadeia de suprimento e, por } \\
\text { fim, em reduções de custos. }\end{array}$ & $\begin{array}{l}\text { Faixa muito estreita } \\
\text { entre os níveis } \\
\text { máximos e mínimos } \\
\text { acordados }\end{array}$ \\
\hline Credibilidade & $\begin{array}{l}\text { Incertezas quanto aos } \\
\text { benefícios potenciais do } \\
\text { VMI }\end{array}$ & $\begin{array}{l}\text { Aumento nos níveis de serviço } \\
\text { e redução de lead times }\end{array}$ & $\begin{array}{l}\text { Relutância entre as } \\
\text { partes, quanto à troca } \\
\text { de informações }\end{array}$ \\
\hline Benevolência & $\begin{array}{l}\text { Possibilidade de vazar } \\
\text { informações cruciais }\end{array}$ & $\begin{array}{l}\text { Garantia de vendas, aumento } \\
\text { na margem de lucro e } \\
\text { diminuição em custos de } \\
\text { transportes }\end{array}$ & $\begin{array}{l}\text { Pouco interesse para } \\
\text { produtos comuns }\end{array}$ \\
\hline Qualidade de TI & $\begin{array}{l}\text { Alto nível de erros e } \\
\text { distorções nas informações }\end{array}$ & $\begin{array}{l}30-40 \% \text { das implementações } \\
\text { VMI atingem grandes } \\
\text { benefícios }\end{array}$ & $\begin{array}{l}\text { Baixa frequência de } \\
\text { interação }\end{array}$ \\
\hline Qualidade da parceria & $\begin{array}{l}\text { Padronização na } \\
\text { identificação dos produtos }\end{array}$ & $\begin{array}{l}\text { Número limitado de associados } \\
\text { estratégicos }\end{array}$ & $\begin{array}{l}\text { Aderência ao VMI por } \\
\text { demanda/exigência do } \\
\text { cliente. }\end{array}$ \\
\hline $\begin{array}{l}\text { Qualidade da informação } \\
\text { Classen } \text { et al. (2008) }\end{array}$ & $\begin{array}{l}\text { Fornecedores exclusivos ou } \\
\text { que possuam tecnologias } \\
\text { críticas }\end{array}$ & $\begin{array}{l}\text { Maior interesse para produtos } \\
\text { estratégicos. }\end{array}$ & \\
\hline
\end{tabular}

Fonte: Silva (2010)

Pode-se perceber no quadro 1 os principais fatores críticos: compartilhamento, disponibilidade, confiabilidade, precisão, facilidade de acesso e consistência das informações. Como barreira, a falta de preparo em conectar informações de demanda, que levam à necessidade de um sistema planejado para o correto funcionamento do VMI. Quanto ao sucesso, o aumento nos níveis de serviço e redução de lead times, além de grandes benefícios apresentados em cerca de 30$40 \%$ dos casos levam à melhoria que a adoção do VMI possibilita.

Segundo Marques et al. (2010), a integração via VMI implica em uma colaboração que une cada processo de planejamento de cada parceiro diferente e leva ao compartilhamento das 
demandas e metas entre os parceiros. Dong et al. (2014) atribuem os benefícios advindos da adoção do VMI a dois fatores: compartilhamento de informação e controle da transferência dessa informação. Choudhary e Shankar (2015) também identificaram o primeiro fator, entre outros, como benefícios, mas sob a condição de uma colaboração efetiva do fornecedor.

Além dos benefícios citados acima há outros, como: a redução de custos; baixos preços no varejo; demanda alta; coordenação ajustada; vantagens nos transportes; elevação de lucros; aumento do nível de serviço ao cliente pelos varejistas; equilíbrio entre oferta e demanda, via redução das incompatibilidades entre elas, que leva a um desempenho superior da cadeia de suprimentos; elevação da frequência de entregas; redução dos níveis de estoque via consolidação das entregas; redução da indisponibilidade de produtos; melhoria no desempenho organizacional; uso mais eficiente das tecnologias de informação e comunicação; redução de custos de distribuição (DARWISH; ODAH, 2010; KANNAN et al., 2013, SETAK; DANESHFAR, 2014, p. 1089; CHEN; WEI, 2012, P. 4396; CHEN et al. 2012; CHOUDHARY; SHANKAR, 2015, p. 68; DONG et al., 2014; NGUGI et al., 2012).

Contudo, Choudhary e Shankar (2015) afirmam que os benefícios dependem da eficiência da emissão do pedido do fornecedor. Caso isso ocorra, o VMI auxilia o fornecedor em: unir as quantidades de ressuprimento em um tamanho de lote econômico em poucas operações e levam à redução de custos em 1,43\%; aumentar o tamanho do inventário e das entregas; reduzir os custos fixos relacionados ao pedido e ao estoque.

Os autores citados acima exemplificam: o aumento da eficiência da emissão do pedido do fornecedor em $30 \%$ leva a redução de custos em 12,2\%, reduz níveis de estoques em 3,67\% e aumenta a frequência do ressuprimento junto ao varejista em 1,8\%. Caso não haja uma integração planejada, pode haver aumentos de custos pelos varejistas na cadeia de suprimentos (CHOUDHARY; SHANKAR, 2015).

Por fim, cabe ressaltar que o VMI é uma mudança no modelo de gestão de estoques, transformando a forma de relacionamento entre parceiros e provendo maior integração nos elos da cadeia de suprimentos.

\section{MATERIAIS E MÉTODOS}

Segundo Halfpenny (1979), em geral, pesquisas qualitativas preocupam-se mais em desenvolver conceitos do que aplicar conceitos já existentes, estudando casos particulares ao invés de populações extensas e descrevendo os significados das ações para os atores. O presente trabalho é caracterizado como uma pesquisa com abordagem qualitativa, com uma pesquisa exploratória e utiliza para isso o método de estudo de caso, para a pesquisa de uma rede varejista do Brasil.

Ao se discutir VMI e estoques, cria-se uma interação com outros temas (MATEEN; CHATERJEE, 2015), como gestão da cadeia de suprimentos. Contudo, nesse estudo foram focadas relações entre eles, em uma rede de varejo de alimentos.

O roteiro teve vinte (20) perguntas, que foram baseadas nos temas revisados pela presente pesquisas, como: logística, cadeia de suprimentos, gerenciamento de estoques, suas práticas e problema e, por fim, o roteiro teve perguntas sobre o VMI. Houve perguntas sobre a relação do VMI e o ECR, mas por questão de foco, esse último foi excluído do artigo.

O roteiro foi enviado por email ao entrevistado, que era Consultor de Sistemas da rede varejista estudada, logo depois ele saiu da empresa. Ele apresentava experiência há mais de 20 anos em Gestão de Sistemas de Informação em redes de varejo do País. A análise foi realizada na 
sistematização de conteúdos da revisão teórica e da pesquisa de campo, unindo-se e contrapondo os principais pontos levantados.

\section{RESULTADOS E DISCUSSÃO}

O objeto deste trabalho é uma das maiores redes de varejo no País, e, no momento da pesquisa, possuía 62 lojas especializadas em eletroeletrônicos, somando $41.229 \mathrm{~m}^{2}$, a qual apresenta excelência no atendimento ao cliente e layout inovador.

De acordo com o entrevistado, o Grupo possui a área de Logística estruturada com o objetivo de atender às necessidades internas, é uma divisão corporativa. Os clientes internos são divididos entre hipermercados, supermercados e minimercados. Há, também, lojas atacadistas de alimentos, de comercialização de eletroeletrônicos e uma área dedicada ao setor de e-commerce, todos caracterizados como clientes.

O gerenciamento dos fornecedores é realizado de acordo com a categoria de produtos. Assim, em mercados grandes (SP) existem CDs especializados em determinados produtos, como por exemplo, mercearia (líquida e seca), frutas, legumes e verduras (FLV), frigorificados e não alimentos. Cada um deles possui seu núcleo de gestão para buscar melhores práticas e processos capazes de otimizar o fluxo inbound e outbound nos CDs e inbound nas lojas.

No gerenciamento de estoques dos produtos nas lojas tudo é informatizado e gerentes e encarregados das seções sabem o volume de produtos nas lojas, tanto dos produtos alocados na gôndola, quanto na retaguarda. O repositor da loja ou do fornecedor não interfere nos níveis de estoque, com exceção nas ações de marketing do fabricante, na adequação da oferta do produto, envolvendo uma negociação específica entre fornecedor e varejista.

Nos CDs existe uma TI que informa diariamente a posição de estoque dos produtos, aqueles que estão perto de atingir o nível de segurança são negociados ou reabastecidos. Segundo o entrevistado, o equilíbrio desse ponto de reabastecimento é o segredo da gestão de estoques da rede. Para ele, o maior problema no gerenciamento de estoques é a cultura de procurar e oferecer ofertas do mercado brasileiro, pois quando há distúrbios em um fluxo na cadeia de suprimentos - via criação de uma nova oferta de produtos - é difícil voltar aos parâmetros anteriores e mais ainda, expurgar as influências desses efeitos no modelo. Isso significa que há uma dificuldade em se balancear a demanda pelo cliente com a configuração que gere ganhos à organização.

Na empresa, o VMI começou a ser discutido na década de 90 e sua utilização ocorre, principalmente, no setor de padarias da empresa, pois os pães industrializados apresentam perecibilidade elevada e necessitam de um processo de logística reversa importante. Essa ferramenta é bastante útil nessa gestão para o fornecedor ter controle real da quantidade de produtos disponíveis no varejo e dados importantes como o vencimento, possibilitando esse controle. $\mathrm{Na}$ utilização do VMI não há uma regra, mas é necessário que os fornecedores da seção estejam aptos a implantá-la de uma maneira mais eficaz ainda que a área de logística do varejista.

A implantação do VMI está relacionada diretamente com a maturidade do fornecedor de cada região, então a rede implantou, a fim de elevar os resultados, práticas de sucesso nas lojas de toda rede. As diferenças que podem existir se relacionam mais ao mix e à quantidade de produtos por loja, pois o VMI é utilizado de forma similar em todas as lojas da rede.

A utilização dessa ferramenta apresenta benefícios, como a eliminação da preocupação com os detalhes das operações de gerenciamento de estoques, pois, uma vez estabelecido um contrato, os resultados de tal fluxo passam a ser cobrados diretamente do fornecedor. Nos casos experimentados 
pelo setor de pães e de FLV, ainda há a vantagem de se eliminar um ciclo inbound-armazenagemoutbound. Além disso, o VMI permite ao varejista menores preocupações acerca da reposição de alguns de seus produtos nas lojas

$\mathrm{O}$ entrevistado não soube informar o número de fornecedores que utilizam o VMI para reposição de estoques nessa rede. As questões relacionadas à avaliação, prévia ou atual, da implantação do VMI e às desvantagens de tal ferramenta também não obtiveram respostas. $\mathrm{O}$ quadro 2 realiza o contraponto das diferenças e das similaridades encontradas na teoria e percebidas na prática da rede varejista de alimentos do estudo de caso:

Quadro 2 - Comparativo entre Teoria e Estudo de Caso

\begin{tabular}{|c|c|c|}
\hline Variável & Teoria & Estudo De Caso \\
\hline Logística & $\begin{array}{l}\text { Promove a integração de informações, } \\
\text { transporte, estoque, armazenamento, } \\
\text { manuseio de materiais e embalagem, para } \\
\text { permitir a alocação correta dos bens na } \\
\text { quantidade e no tempo requerido e ao } \\
\text { menor custo. }\end{array}$ & $\begin{array}{l}\text { Uma divisão corporativa que deve atender as } \\
\text { necessidades de toda a rede, provendo, } \\
\text { portanto, sua integração. }\end{array}$ \\
\hline $\begin{array}{l}\text { Gerenciamento } \\
\text { de Estoque }\end{array}$ & $\begin{array}{l}\text { É um ponto crucial para o funcionamento } \\
\text { aprimorado da logística e da cadeia de } \\
\text { suprimentos em geral, na disponibilização } \\
\text { dos produtos no tempo e na qualidade } \\
\text { desejadas pelo cliente. }\end{array}$ & $\begin{array}{l}\text { Para que o gerenciamento de estoques dos } \\
\text { produtos seja otimizada, é utilizado o VMI, não } \\
\text { há autonomia da loja na determinação de seus } \\
\text { níveis de estoque. }\end{array}$ \\
\hline VMI & $\begin{array}{l}\text { Ferramenta na qual o fornecedor controla } \\
\text { os níveis de estoque de seus clientes e } \\
\text { decide quando e quanto entregar de } \\
\text { mercadoria para cada cliente. }\end{array}$ & $\begin{array}{l}\text { Os fornecedores da Padaria e do FLV tem } \\
\text { acesso direto ao nível de estoque dos produtos } \\
\text { fornecidos, responsabilizando-se pelo } \\
\text { reabastecimento nas lojas e recolhimento de } \\
\text { produtos vencidos/estragados. }\end{array}$ \\
\hline $\begin{array}{l}\text { VMI } \\
\text { (vantagens) }\end{array}$ & $\begin{array}{l}\text { Diminuição de perdas, ganhos de } \\
\text { eficiência, redução de rupturas e custos, } \\
\text { rapidez, confiabilidade e capacidade de } \\
\text { rastreabilidade. }\end{array}$ & $\begin{array}{l}\text { Detalhes da operação de estoques deixam de } \\
\text { ser preocupação do cliente para ser gerido pelo } \\
\text { fornecedor, nos setores de Padaria e FLV se } \\
\text { elimina um ciclo inbound-armazenagem- } \\
\text { outbound. }\end{array}$ \\
\hline $\begin{array}{l}\text { VMI } \\
\text { (desvantagens) }\end{array}$ & Custos elevados de implantação. & $\begin{array}{l}\text { Sua implantação depende da maturidade do } \\
\text { fornecedor, o que impossibilita a ampliação da } \\
\text { utilização .de tal ferramenta. }\end{array}$ \\
\hline
\end{tabular}

Fonte: Elaborada pelos autores 


\section{CONCLUSÃO}

O maior desafio de se gerenciar estoques é monitorar a quantidade de itens distintos dentro de um CD. No auxílio a transpor essa barreira, o VMI permite que as operações do setor ocorram com um maior compartilhamento de informações, com o fornecedor se responsabilizando pelo gerenciamento dos níveis de estoque de seus clientes.

$\mathrm{Na}$ rede varejista pesquisada, a área de Logística está consolidada e é corporativa, respondendo diretamente às solicitações de todas as lojas da rede e permitindo uma unidade na implantação em todas as lojas da rede das melhores práticas adotadas. Isso resulta em uma prestação de serviço de qualidade elevada aos clientes.

Como a utilização do VMI permite a visualização diária das posições de estoques dos produtos nos CDs da rede, o gerenciamento dos estoques da rede possibilita que os produtos estejam disponíveis para as solicitações das lojas e não se esgotem.

Há informatização dos estoques nas lojas, com seu reabastecimento realizado através do CD ou diretamente do fornecedor - como no caso dos setores que utilizam o VMI, ao ser atingido o nível de estoque mínimo. Assim, tem-se um melhor atendimento ao cliente, que passa a ter a garantia da disponibilidade do produto na loja, e a redução dos custos da operação. Essa redução é favorecida pelo controle diário dos produtos vendidos, evitando alocação de grandes espaços nos estoques.

O VMI permitiu aos fornecedores, com destaque para os setores de Pães e FLV, o controle direto dos produtos comercializados nas lojas. Apesar do custo elevado, a implantação é justificada pelo resultado na satisfação do cliente alta, menores preocupações com o gerenciamento dos estoques do produto e uma considerável redução de estoques.

Um fator limitante observado na pesquisa para a expansão dessa ferramenta relaciona-se com a maturidade dos fornecedores, que devem possuir autonomia e conhecimentos suficientes para assumir o controle direto dos estoques de seu cliente. Devido a essa falta de conhecimento, havia o receio em se comprometer com o gerenciamento direto do estoque do cliente, pois ela pode se mostrar mais complicada que o esperado, gerar gastos desnecessários, caso não seja implantada corretamente.

Ao se superar os obstáculos iniciais de implantação do VMI, que podem ser minimizados por meio de uma pesquisa com fornecedores e demonstração a eles, a fim de se implantar o VMI no varejo supermercadista brasileiro de grande porte.

O VMI possibilita uma redução de custos ainda maior no setor, já que através da ampla utilização da ferramenta, ocorrerão melhorias no gerenciamento de estoques, nas relações entre os parceiros da cadeia de suprimentos, e no atendimento ao cliente. Para isso, é necessário o comprometimento dos fornecedores para assumirem esse controle e compromisso com seus clientes da cadeia de suprimentos.

\section{REFERENCIAS}

AMIRJABBARI, Bahareh; BHUIYAN, Nadia. Determining supply chain safety stock level and location. Journal of Industrial Engineering and Management, v. 10, n. 1, p. 42-71, 2014.

BALLOU, Ronald H. Gerenciamento da cadeia de suprimentos/logística empresarial. Porto Alegre: Bookman, 2006.

BERTAGLIA, Paulo Roberto. Logística e gerenciamento da cadeia de abastecimento. $2^{\text {a }}$ Ed. São Paulo: Saraiva, 2009. 
BOWERSOX, Donald J.; CLOSS, David J.; COOPER, Martha B. Gestão da Cadeia de Suprimentos e Logística. Rio de Janeiro: Elsevier, 2007

BRAGLIA, Marcello; CASTELLANO, Davide; FROSOLINI, Marco. Safety stock management in single vendorsingle buyer problem under VMI with consignment stock agreement. International Journal of Production Economics, v. 154, p. 16-31, 2014.

CHEN, Xu; Hao, Gang; Li, Xun ;Yiu, Ka Fai Cedric. The impact of demand variability and transshipment on vendor's distribution policies under vendor managed inventory strategy. International Journal of Production Economics, v. 139, n. 1, p. 42-48, 2012.

CHEN, Liang-Tu. Dynamic supply chain coordination under consignment and vendor-managed inventory in retailercentric B2B electronic markets. Industrial Marketing Management, v. 42, n. 4, p. 518-531, 2013.

CHEN, Liang-Tu; WEI, Chun-Chin. Multi-period channel coordination in vendor-managed inventory for deteriorating goods. International Journal of Production Research, v. 50, n. 16, p. 4396-4413, 2012.

CHOUDHARY, Devendra; SHANKAR, Ravi. The value of VMI beyond information sharing in a single supplier multiple retailers supply chain under a non-stationary (R n, S n) policy. Omega, v. 51, p. 59-70, 2015.

DARWISH, Mohammed Abdulaziz; ODAH, O. M. Vendor managed inventory model for single-vendor multi-retailer supply chains. European Journal of Operational Research, v. 204, n. 3, p. 473-484, 2010.

DONG, Yan; DRESNER, Martin; YAO, Yuliang. Beyond Information Sharing: An Empirical Analysis of Vendor-Managed Inventory. Production and Operations Management, v. 23, n. 5, p. 817-828, 2014.

GIANESI, Irineu Gustavo Nogueira; DE BIAZZI, Jorge Luiz. Gestão estratégica dos estoques. Revista de Administração, v. 46, n. 3, p. 290-304, 2011.

GOVINDAN, Kannan; GRIGORE, Maria Christina; KANNAN, Devika; KUMAR, A. Senthil An analysis of the general benefits of a centralized VMI system based on the EOQ model. International Journal of Production Research, v. 51, n. 1, p. 172-188, 2013.

HALFPENNY, Peter. The Analysis of Qualitative Data. California: The Sociological Review, 1979.

MARQUÈS, Guillaume; THIERRY, Caroline, LAMOTHE, Jacques; GOURC, Didier. A review of Vendor Managed Inventory (VMI): from concept to processes. Production Planning \& Control, v. 21, n. 6, p. 547-561, 2010.

MATEEN, Arqum; CHATTERJEE, Ashis Kumar; MITRA, Subrata. VMI for single-vendor multi-retailer supply chains under stochastic demand. Computers \& Industrial Engineering, v. 79, p. 95-102, 2015.

NGUGI, John Karanja; KIPKOGEI, Jonah Aiyabei; MAROKO, Phillip Manyura; NGUGI, Patrick Karanja. Influence of vendor inventory management on organizational performance in retail outlets in Kenya: A case of Uchumi supermarkets. International Journal of Business and Social Research, v. 2, n. 7, p. 62-69, 2012.

OLIVEIRA, Lindomar Subtil; HATAKEYAMA, Kazuo. Um estudo sobre a implantação de sistemas ERP: pesquisa realizada em grandes empresas industriais. Revista Produção, v.22, n.3, p. 596-611, 2012.

REGOA, José Roberto do; MESQUITA, Marco Aurélio de. Controle de estoque de peças de reposição: uma revisão da literatura. Revista Produção, São Paulo, v. 21, n.4, p. 645-655, out./ dez. 2011.

SANTOS, M. G. Abordagem sobre a aplicabilidade da Tecnologia RFID na Cadeia de Suprimentos e na Administração de Estoques. Dissertação (Tecnólogo). 78 p. Faculdades de Tecnologia de São Paulo. Centro Tecnológico da Zona Leste. São Paulo, 2009.

SANTOS, Antônio Marcos dos; RODRIGUES, Iana Araújo. Controle de Estoque de Materiais com Diferentes Padrões de Demanda: Estudo de Caso de uma Indústria Química. Gestão e Produção. v.13, n.2, p.223-231, 2006.

SETAK, Mustafá; DANESHFAR, L. An Inventory Model for Deteriorating Items Using Vendor-Managed Inventory Policy. International Journal of Engineering-Transactions A: Basics, v. 27, n. 7, p. 1081-1090, 2014.

SGARBI JUNIOR, Guilherme; CARDOSO, Álvaro Azevedo Lean Seis Sigma na Logística - Aplicação na Gestão dos Estoques em uma Empresa de Autopeças. In: SIMPÓSIO DE EXCELÊNCIA EM GESTÃO E TECNOLOGIA, 7. 2011, Resende, Rio de Janeiro. Anais... Rio de Janeiro, 2011.

SILVA, Gabriel Rossoni Desenvolvimento de um modelo de simulação para avaliação do desempenho de uma cadeia de suprimentos do ramo de mineração através da adoção da parceria VMI (Vendor Managed Inventory). 
Dissertação (Mestrado Interdepartamental em Engenharia de Sistemas Logísticos), Universidade de São Paulo. Escola Politécnica da USP. 218 p. 2010.

SILVA, Thiago Gomes da; BARBOZA, Fabiano; MARUJO, Lino G. Análise dos Impactos das Diferentes Políticas de Gestão dos Estoques de Suprimentos em uma Empresa do Setor de Bebidas Através de Simulação. Revista Gestão
Industrial,
Paraná,
v. $4, \quad$ n.2,
p. 34-75,
2008.
Disponível
em:

revistas.utfpr.edu.br/pg/index.php/revistagi/article/view/190. Acesso em: 09 mai. 2012.

VASCONCELOS, Thaís. Furtado; NOGUEIRA, Cíntia Carvalho; MELO, André Cristiano Silva. A importância da estruturação de sistemas de medição de desempenho para o monitoramento da gestão de estoques na indústria de bebidas. In: ENCONTRO NACIONAL DE ENGENHARIA DE PRODUÇÃO, 31., 2011, Belo Horizonte. Anais... Belo Horizonte: Associação Brasileira de Engenharia de Produção, 2011. 